IMMUNOGENETICS

\title{
Modulating lymphocyte development
}

MicroRNAs (miRNAs) are small, $\sim 22$ nucleotide, RNAs that have been shown to have a role in regulating gene expression during development in Caenorhabditis elegans and Drosophila. Although mammalian miRNAs have been identified, no function had been ascribed to them until this study, in Science, identified mouse miRNAs as regulators of haematopoiesis.

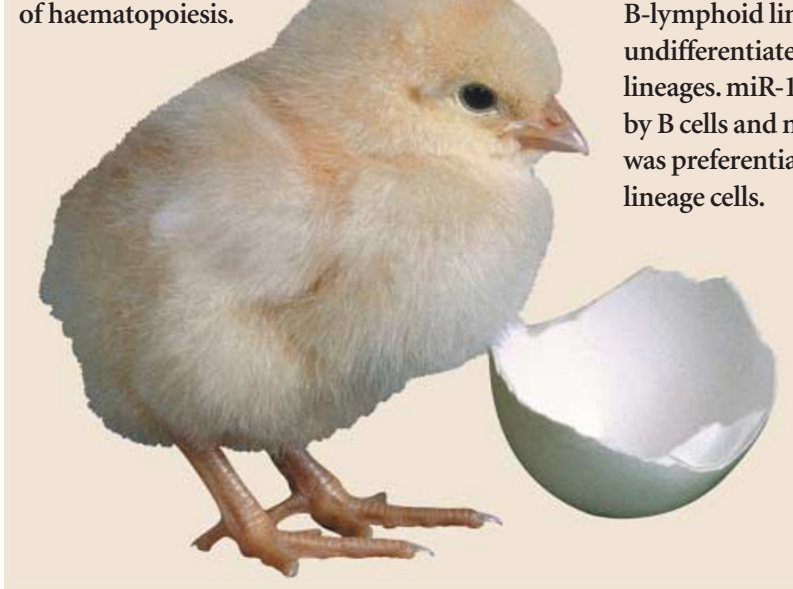

To investigate the importance of mammalian miRNAs in haematopoietic-cell development, Chen et al. cloned several miRNAs from mouse bone marrow, three of which — miR-181, miR-223 and miR-142 showed preferential expression in haematopoietic tissue. Further analysis indicated that in the bone marrow, miR-181 was preferentially expressed by the B-lymphoid lineage compared with undifferentiated progenitor cells or other lineages. miR-142 was most highly expressed by $B$ cells and myeloid cells, whereas miR-223 was preferentially expressed by myeloid lineage cells.

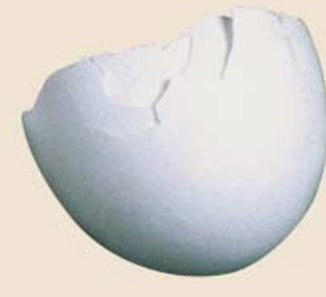

Can miRNAs regulate haematopoietic lineage commitment? Ectopic expression of miR- 181 by undifferentiated progenitor cells from the bone marrow led to a selective increase in the number of B-lineage cells after in vitro culture, whereas miR-142 and miR-223 expression markedly enhanced the proportion of T-lineage cells. Further evidence of a role for miR-181 in B-lineage differentiation was provided by the observation that transfer of cells expressing miR-181 to lethally irradiated recipients led to a marked increase in the proportion of B-lineage cells in the peripheral blood and a concomitant decrease in the T-cell population, in particular among the $\mathrm{CD} 8^{+} \mathrm{T}$-cell subset.

This study provides the first demonstration that miRNAs have a role in regulating development in mammals. Interestingly, although these miRNAs influenced the development of specific lymphoid populations, they did not completely block differentiation to other lineages, leading the authors to suggest that these individual miRNAs act as lineage modulators rather than as on/off switches.

(i) References and links Karen Honey ORIGINAL RESEARCH PAPER Chen, C. Z. et al. MicroRNAs modulate hematopoietic lineage differentiation. Science 303, 83-86 (2004)

\section{Regulating lipid presentation}

Although it is well established that the nonclassical MHC class-I-like molecule Cd1d presents lipid antigens to T cells, little is known about the mechanisms that are involved in lipid loading. A recent study in Science now reports a role for lipid transfer proteins (LTPs), specifically saposins, in editing Cd1d-bound lipids in vivo.

A large proportion of Cd1d-restricted T cells, known as $\mathrm{V} \alpha 14$ natural killer $\mathrm{T}$ (NKT) cells, express a semi-invariant Vo14-J $\alpha 18$ T-cell receptor $\alpha$-chain and recognize the natural ligand mimic, the marine-spongederived glycolipid $\alpha$-galactosyl ceramide $(\alpha-\mathrm{GC})$. Recognition of endogenous Cd1d-bound ligands by $\mathrm{V} \alpha 14 \mathrm{NKT}$ cells requires Cd1d trafficking through the endosomal pathway, and so the authors set out to investigate whether endosomal proteins involved in lipid metabolism influence Cd1d antigen presentation.

Saposins A, B, C and D are regulators of endosomal lipid metabolism generated by proteolysis of a precursor - prosaposin. Using $\alpha$-GC-loaded Cd1d tetramers, Zhou et al. observed that despite expressing normal levels of Cd1d on the surface of splenocytes and thymocytes, prosaposin-deficient mice lacked V $\alpha 14$ NKT cells. Other lymphocyte populations were unaffected by the deficiency, indicating that prosaposin specifically affects V $\alpha 14$ NKT cells.

Analysis of the ability of prosaposindeficient thymocytes to stimulate a panel of Cd1d-restricted T-cell hybridomas showed that saposins are specifically involved in Cd1d presentation of endogenous V $\alpha 14$ NKT-cell ligands. No global defect of endosome function was detected, and further evidence of a specific role for saposins in regulating $\mathrm{Cd} 1 \mathrm{~d}$ presentation was provided by the observation that prosaposin and Cd1d co-localized with lysosome-associated membrane protein 1 (Lamp1) — a marker for late endosomes.

The mechanism by which saposins regulate Cd1d presentation was assessed in a cell-free system. Each saposin protein promoted the exchange of Cd1d-bound acidic trisialoganglioside for liposome containing phosphatidylserine, albeit with differing efficiencies. In addition, a direct interaction was detected between saposin A and Cd1d complexed with some lipids, but not others, indicating that the interaction might depend on the lipid head group and might provide some specificity for the lipids loaded by the individual saposins.

This study identifies saposins as crucial components in regulating Cd1d presentation of the endogenous lipid ligands that are responsible for Vo14 NKT-cell selection in vivo. However, it remains to be confirmed whether the in vitro results translate into the molecular mechanism by which saposins mediate this effect in vivo.

(2) References and links

Karen Honey

ORIGINAL RESEARCH PAPER Zhou, D. et al. Editing of Cd1d-

bound lipid antigens by endosomal lipid transfer proteins. Science 18 December 2003 (doi: 10.1126/science.1092009) FURTHER READING Moody, D. B. \& Porcelli, S. A. Intracellular pathways of CD1 antigen presentation. Nature Rev. Immunol. 3, 11-22 (2003) 